April 2018

\title{
Myocardial perfusion imaging: the gatekeeper and advisor
}

Maseeh uz Zaman

Aga Khan University, maseeh.uzzaman@aku.edu

Nosheen Fatima

Aga Khan University, nosheen.fatima@aku.edu

Follow this and additional works at: https://ecommons.aku.edu/pakistan_fhs_mc_radiol

Part of the Radiology Commons

\section{Recommended Citation}

uz Zaman, M., Fatima, N. (2018). Myocardial perfusion imaging: the gatekeeper and advisor. Journal of the College of Physicians and Surgeons--Pakistan, 28(4), 259-261.

Available at: https://ecommons.aku.edu/pakistan_fhs_mc_radiol/115 


\title{
Myocardial Perfusion Imaging: The Gatekeeper and Advisor
}

\author{
Maseeh uz Zaman and Nosheen Fatima
}

Radiation-based morphological and functional imagings, like radiology and nuclear medicine, are considered the standard of care in modern medicine. Nuclear cardiology is one of the most important domains of nuclear medicine which has been playing an important role in the diagnosis, prognosis, and decision-making in clinical cardiology. Myocardial perfusion imagings (MPIs), using single-photon emission computerised tomography (SPECT) and positron emission tomography (PET), are the most commonly performed nuclear cardiology procedures worldwide. Reasons for MPI's unprecedented popularity are its ability to study myocardial perfusion non-invasively, precise estimation of left ventricular function, myocardial viability and risk stratification in patients with known coronary artery disease (CAD) or after myocardial infarction. Multigated acquisition (MUGA) imaging is considered as the modality of choice for precise assessment of left ventricular ejection fraction (LVEF) and volumes. But echocardiogram, being the major competitor, is leading far ahead in the clinical practice. Similarly, radionuclide first passed radionuclide angiogram (FPRA) is considered as the gold standard for precise estimation of right ventricular ejection fraction (RVEF) and cardiac shunt quantification, but could not get popularity.

Over the last four decades, MPI has become a major non-invasive tool for evaluation of CAD. ${ }^{1}$ However, the greatest strength of MPI is due to its established role in risk stratification in patients with known CAD or postmyocardial infarction. ${ }^{2}$ Precise assessment of myocardial blood flow (MBF), using PET or SPECT methodology, has made MPI an indispensable tool for evaluation and management of CAD. ${ }^{3}$ The most popular indication of $\mathrm{MPI}$ is its role as the gatekeeper to catheterisation laboratory for selection of the patients who need invasive approach or would require conservative management. Other important indications of $\mathrm{MPI}$ are the diagnosis of CAD in patients with intermediate pretest probability or asymptomatic patients with abnormal ECG or symptomatic patient with normal ECG. MPI is also indicated for assessment of

Department of Radiology, Section of NM and PET/CT Imaging Services, The Aga Khan University Hospital, Karachi.

Correspondence: Prof. Maseeh uz Zaman, Department of Radiology, Section of NM and PET/CT Imaging Services, The Aga Khan University Hospital, Stadium Road, Karachi. E-mail: maseeh.uzzaman@aku.edu

Received: November 17, 2017; Accepted: December 03, 2017. efficacy of therapeutic interventions like angioplasty or coronary artery bypass graft (CABG) surgery, risk stratification, and preoperative cardiac assessment in intermediate to high risk patients. Quantification of coronary flow reserve (CFR), using PET or SPECT, provides an important piece of clinical information about the hemodynamic significance of epicardial coronary lesion or diagnosis of small vessel disease. ${ }^{1}$ Recently, plaque imaging using PET-MPI has opened new avenue for detection of vulnerable plaques, and targeted therapy of these plaque is expected to reduce CAD related morbidity and mortality. 4

Radiopharmaceuticals, which are used for MPI, include Tc-99m labelled Methoxy IsoButyl Isonitrile (MIBI), Tetrofosmin (MYOVIEW), BATO and NOET compounds. Last two agents have not yet approved for clinical use so far. For assessment of the myocardial viability, Thallium201 (TI-201) is more sensitive and specific, as presence of redistribution is a reliable indicator of intact cell membrane and cell viability. However, due to its cost and significantly higher radiation exposure, various centres use Tc-99m MIBI and MYOVIEW with sublingual nitrate augmentation with reasonably good diagnostic accuracy. ${ }^{5}$

MPI is performed to assess the LV myocardial perfusion and function during stress and at rest. For stress part, dynamic exercise on treadmill or ergometer using Bruce or Modified Bruce protocol is a preferred choice as it is more physiological and provides important information about the functional capacity like Metabolic Equivalent (METS), which has an established prognostic value for future cardiac events. For patients who are unable to perform exercise due to physical limitation or unreadable ECGs like left bundle branch block (LBBB) or WolffeParkinson-White (WPW) syndrome, vasodilator infusion like adenosine, dipyridamole or regadenoson are the alternatives. However, these are not indicated if patient has history of bronchospasm or asthma as these agents may induce severe bronchospasm (less likely with regadenoson). Vasodilator stress may be used with low level exercise (hybrid protocol) as this modification reduces the side effects like headache and hypotension; and also increases the sensitivity of test as well. Tc-99m MIBI or MYOVIEW are injected intravenously at peak stress and during resting status as these agents distribute according to regional perfusion status at the time of injection and gets fixed into myocardium and provides a snap-shot images of LV perfusion later on. Stress and rest MPIs may be performed on two separate days (average 1.5 hour/day) or the same day (average 
3-4 hours). Gated SPECT images are acquired under a gamma camera, which needs 15-20 minutes for acquisition of complete set of data. After reconstruction, images are analysed visually and semi-quantitatively using 17 segment model of American Heart Association (AHA) for assessment of LV perfusion, function, wall motion abnormality and thickness. ${ }^{6}$

Based on MPI findings, patients are categorised as low $(<1 \%)$, intermediate $(1-3 \%)$, and high $(>3 \%)$ risk groups for probability of annual major acute cardiac events (MACE) like fatal or non-fatal myocardial infarction. A normal Gated MPI (GMPI) has a negative predictive value (NPV) of $>99 \%$ with an established $0.4-0.6 \%$ annual incidence of MACE for exercise GMPI. However, NPV of vasodilator stress GMPI is relatively low as compared to exercise GMPI.7 Various management strategies have been formulated based on perfusion and functional parameters like LVEF and end systolic volume (ESV) derived from GMPI. Patients with LVEF $\geq 50 \%$, without or with mild to moderate perfusion defects, are considered low risk and deserve conservative management; but those with LVEF $30-50 \%$ with mild to moderate perfusion defects are considered intermediate risk and are considered candidates for revascularisation. Patients with LVEF $<30 \%$ are considered at high risk and subjected to viability study with Thallium-201 or MIBI or MYOVIEW with nitrate augmentation to confirm presence of hibernating myocardium. Presence or absence of hibernating myocardium guides the treating cardiologist about revascularisation or conservative management, respectively. Similarly, patients with LVEF $<45 \%$ and ESV $\leq 70 \mathrm{ml}$ or LVEF $<45 \%$ and ESV $>70 \mathrm{ml}$ are considered to have lower and higher annual mortality rates, respectively. 8

In recent years $\mathrm{PET} / \mathrm{CT}$ has also stepped in from oncology into clinical cardiology domain. Being a hybrid imaging modality, it provides information regarding perfusion, function, coronary artery calcium (CAC), coronary anatomy, and precise estimation of CFR in one session. This one-stop-shop solution with lesser time and lesser radiation exposure are the real driving forces favouring the robust growth of PET/CT MPI. However, availability of PET/CT facilities and its cost are the major antagonistic factors. 9

Over the last four decades, there has been a tremendous increase in utilisation of SPECT-MPI all over the world. High sensitivity, non-invasiveness, and relatively easy availability are the major factors responsible for this positive worldwide trend. However, this trend has also contributed significantly high radiation exposure to general public. According to a report, radiation exposure from SPECT-MPI has increased from $1 \%$ to $10.5 \%$ of total exposure over the last 35 years to US population. Similarly, according to National Council for Radiation Protection and Management (NCRP), over the last 30 years there has been six-fold increase in radiation exposure to the American population. ${ }^{10}$ Stochastic effects of radiation, like malignancy or genetic mutations, are the primary concerns which are unfortunately less understood by medical community. To address these issues, International Atomic Energy Agency (IAEA) has introduced strategy of justification and optimisation for radiation-based procedures. Similarly, American Society of Nuclear Cardiology (ASNC) has introduced appropriate use criteria (AUC) for MPI in an attempt to curtail unjustified MPIs contributing to radiation exposure to general public. Various professional societies of US and Europe have also released radiation dose reducing guidelines like use of stress-only protocol, use of low doses of radioisotope, use of more sensitive gamma cameras, use of better reconstructive algorithm and preference of PET-MPI over SPECT-MPI, when available. ${ }^{1}$

MPI using SPECT or PET is the most sensitive functional imaging modality for precise assessment of myocardial perfusion and function. Being a non-invasive tool, it guides the cardiologists about the presence of functionally significant $C A D$, selection of appropriate treatment strategies, and most importantly risk stratification in patients with or without a major cardiac event. SPECT-MPI has been used overwhelmingly worldwide in the last four decades, which has made it one of the major contributors of radiation exposure to general public. Stochastic effects, like cancer and genetic mutations, are the major concerns which are unfortunately less understood. To address this important aspect, various strategies have been formulated by IAEA; and various appropriate use criteria have been released by professional societies of the US and Europe. Recently, PET-MPI has been promoted over SPECT-MPI due to its higher sensitivity, diagnostic accuracy, and absolute quantification of various functional parameters in shorter time with significantly lower radiation exposures.

\section{REFERENCES}

1. Dorbala S, Blankstein R, Skali H, Park M, Fantony J, Mauceri C, et al. Approaches to reducing radiation dose from radionuclide myocardial perfusion imaging. J Nucl Med 2015; 56:592-9.

2. Shaw L, Narula J. Risk assessment and predictive value of coronary artery disease testing. J Nucl Med 2009; 50:12961306.

3. Naya M, Murthy V, Taqueti V, Foster C, Klein J, Garber M, et al. Preserved coronary flow reserve effectively excludes high-risk coronary artery disease on angiography. J Nucl Med 2014; 55: 248-55.

4. Evans N, Tarkin J, Chowdhury M, Warburton E, Rudd J. PET imaging of atherosclerotic disease: advancing plaque assessment from anatomy to pathophysiology. Curr Atheroscler Rep 2016 18:30 (e-pub).

5. Niyaz K, Niaz K, Kamal S, Husssain W, Zaman M, Habib S, et al. Assessment of viable myocardium by nitrate augmented 
99mTc MIBI myocardial perfusion imaging. J Pak Med Assoc 2007; 57:83-7.

6. Klocke F, Baird M, Lorell B, Gibbons G. ACC/AHA/ASNC Guidelines for the clinical use of cardiac radionuclide imagingexecutive summary: a report of the American College of Cardiology/American Heart Association Task Force on Practice Guidelines (ACC/AHA/ASNC Committee to Revise the 1995 Guidelines for the clinical use of cardiac radionuclide imaging). J Am Coll Cardiol 2003; 42:1318-33.

7. Fatima N, Zaman M. Higher event rate in Normal GMPI using dipyridamole: Run for reliability. Ind J Nulc Med 2012; 2:172-5.
8. Sharir T, Germano G, Kang X, Lewin H, Miranda H, Cohen I, et al. Prediction of myocardial infarction versus cardiac death by gated myocardial perfusion SPECT: Risk stratification by the amount of stress-induced ischemia and the post-stress ejection fraction. J Nucl Med 2001; 42:831-7.

9. Ghotbi A, Kjær A, Hasbak P. Comparison of PET rubidium-82 with conventional SPECT myocardial perfusion imaging. Clin Physiol Funct Imag 2014; 34:163-70.

10. Bolus N. NCRP report 160 and what it means for medical imaging and nuclear medicine. J Nucl Med Technol 2013; 41:1-6. 